\title{
LUMBAGO, UNA MIRADA ERGONÓMICA
}

\author{
LUMBAR PAIN, AN ERGONOMIC APPROACH
}

\section{Giancarlo Roma Chahud*}

\begin{abstract}
Resumen: El lumbago es un síntoma común experimentado por individuos de todas las edades, siendo actualmente la principal causa mundial de incapacidad. Los mayores incrementos de incidencia en las últimas décadas ocurrieron en países de ingresos medios y bajos con un impacto económico relevante, constituyendo una preocupación global y urgente de salud pública. Genera los costos directos más altos por enfermedades musculoesqueléticas en la industria. Personas con trabajos demandantes, comorbilidades físicas y/o psíquicas tienen un riesgo significativamente más alto de desarrollarlo. La mayoría de los pacientes se recupera rápido de un primer episodio y pese a que las recurrencias son frecuentes una pequeña fracción migra a la cronicidad. En una gran proporción de los casos no se puede diagnosticar una causa precisa. El tratamiento recomendado del lumbago inespecífico inicialmente tiene un enfoque biopsicosocial con herramientas educativas, conductuales, psicológicas y ergonómicas, evitando imágenes, fármacos y otros procedimientos médicos; pese a ello se verifican importantes diferencias entre las recomendaciones formales y lo realizado en la práctica, situación que deteriora su pronóstico e incrementa costos. Soluciones prometedoras son la implementación efectiva de las mejores prácticas clínicas, integrar los sistemas sanitarios al cuidado ocupacional e incorporar la ergonomía de forma anticipativa, sistémica y participativa. El objetivo de esta revisión es presentar una mirada actualizada del lumbago desde el punto de vista ergonómico dada su alta incidencia e impacto sobre las personas y organizaciones. La búsqueda bibliográfica se realizó por medio de bases de datos de revistas científicas, utilizando como criterios de inclusión ergonomía y lumbago.
\end{abstract}

Palabras clave: Lumbago, ergonomía, musculoesquelético, factores de riesgo.

\begin{abstract}
Low back pain is a common symptom experienced by individuals of all ages, and is currently the world's leading cause of disability. The greatest increases in incidence in recent decades occurred in low and middle income countries with a significant economic impact, constituting a global and urgent public health concern. It generates the highest direct costs for musculoskeletal diseases in the industry. People with demanding jobs, physical and / or mental comorbidities have a significantly higher risk of developing it. Most patients recover quickly from a first episode and despite recurrences being frequent, a small fraction migrates to chronicity. In a large proportion of cases, a precise cause cannot be diagnosed. The recommended treatment for nonspecific low back pain initially has a biopsychosocial approach with educational, behavioral, psychological and ergonomic tools, avoiding images, drugs and other medical procedures; despite this, there are important differences between the formal recommendations and what has been done in practice, a situation that deteriorates its prognosis and increases costs. Promising solutions are the effective implementation of best clinical practices, integrating health systems into occupational care, and incorporating ergonomics in an anticipatory, systemic, and participatory way. The objective of this review is to present an updated look
\end{abstract}

"Investigador independiente. Santiago, Chile. Correo electrónico: dr.giancarloroma@gmail.com. Orcid: https://orcid.org/oooo-0003-0642-8182 
of low back pain from an ergonomic point of view given its high incidence and impact on people and organizations. The bibliographic search was carried out through databases of scientific journals, using ergonomics and low back pain as inclusion criterio.

Keywords: Low back pain, ergonomics, musculoskeletal, risk factors.

Recepción: 07.10.2021 / Revisión: 08.11.2021 / Aceptación: 03.12.2021

\section{Introducción}

El lumbago es un síntoma común, experimentado por gente de todo grupo etario (figura 1), afectando hasta el $84 \%$ de los adultos en algún momento de su vida (Brisby, 2016; Cassidy et al., 1998; Deyo \& Tsui-Wu, 1987).

Figura 1. Prevalencia media de lumbago por género y edad.

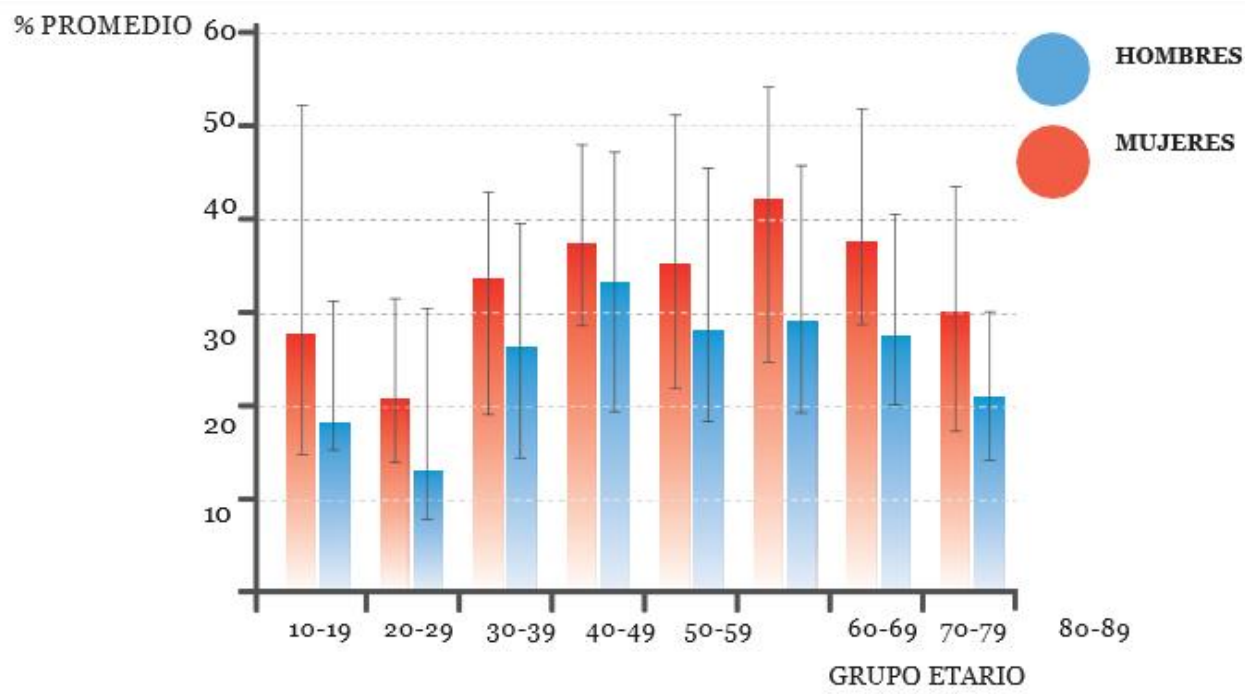

Fuente. Adaptado de Low Back Pain 1 (Lancet, 2018).

Es la causa más común de consultas médicas (Global Burden of Disease, [GBD], 2015) afectando 540 millones de personas en 2015, llegando a ser la principal causa de incapacidad a nivel global. Los mayores incrementos de incidencia en las últimas décadas han ocurrido en países de ingresos bajos y medios incluyendo naciones de Asia, África y Medio Oriente, donde los sistemas de salud y seguridad social son débiles, generando un impacto económico relevante (Hartvigsen \& Hancock, 2018; Maniadakis \& Gray, 2000). Es un motivo frecuente de consulta en unidades de urgencia (Coombs et al., 2021). En Estados Unidos la industria estima en 149 millones los días perdidos al año por lumbago, teniendo un costo anual que excede los 100 billones de dólares; dos tercios de ese valor se debe a salarios disminuidos y pérdidas de productividad, el resto a costos directos por exámenes, medicamentos e intervenciones (LBPSWG, 2018; Katz, 2006; Guo et al., 1999), superando todos los costos directos de los demás trastornos musculoesqueléticos (WHO, 2021; Anon, 2013). Entre 1990 y 2016 en EE. UU. hubo un alza de 2,4 a 3,0 millones de años con discapacidad por lumbago (The US Burden of Disease Collaborators, 2018). A nivel mundial en 2015 se generaron 60 
millones de años de incapacidad por lumbago, representando un ascenso del $54 \%$ respecto a 1990 (figura 2).

Figura 2. Carga global por lumbago, ajustado en años perdidos por discapacidad, por grupo etario para 1990 y 2015.

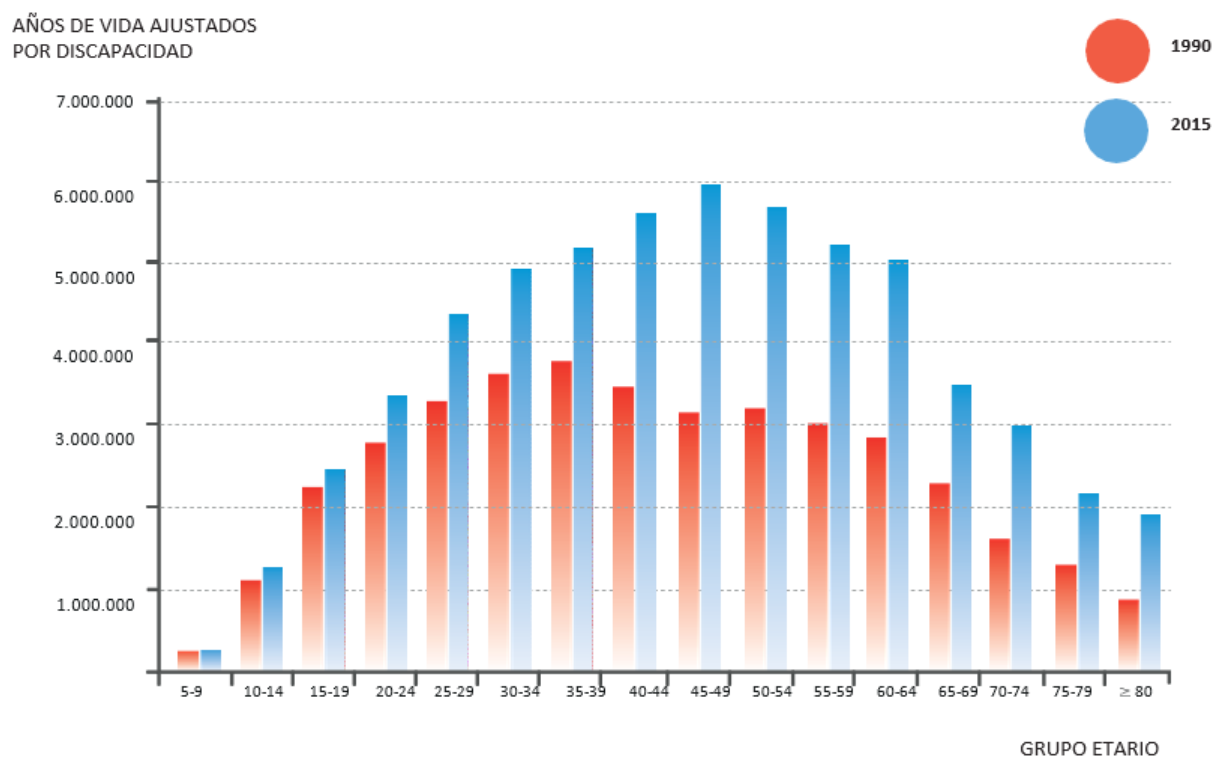

Fuente. Adaptado del informe Global Health Data Exchange (2021).

Factores de riesgo como antecedentes mórbidos personales, psicosociales, genéticos y ocupacionales se asocian con alta prevalencia de lumbago; el control de estos factores más un adecuado diseño y organización del trabajo, junto al uso de dispositivos de asistencia de bajo costo, son favorables en la reducción de su incidencia (Muslim \& Nussbaum, 2017). Factores pronósticos de recaída incluyen exposición a posturas incómodas, largos períodos sentado, más de 2 episodios previos (Da Silva et al., 2019) y factores psicosociales (Lardon et al., 2018; Vranceanu et al., 2009). Su recurrencia es común, con 69\% de recidivas dentro de los 12 meses siguientes a su recuperación inicial, limitando actividades y requiriendo asistencia sanitaria. Pese a la gran cantidad de tratamientos disponibles y recursos destinados a su manejo, el número de individuos afectados y sus costos siguen incrementándose (The US Burden of Disease Collaborators, 2018; Hoy et al., 2010; Freburger et al., 2009).

Vivir con lumbago es una carga significativa para las personas que afecta muchos aspectos de sus vidas, generando múltiples desafíos vitales y profundas consecuencias psicosociales (Blach, 2021).

Guías clínicas de países desarrollados como Estados Unidos (Qaseem et al., 2017), Reino Unido (UK National Institute for Health and Care Excellence [UKNIH\&CS], 2016) o Dinamarca (Stochkendahl et al., 2018), recomiendan aproximaciones similares para su tratamiento, inicialmente sin fármacos, incluyendo el uso de un marco referencial biopsicosocial, educación, automanejo, reincorporación a las actividades normales, ejercicios y programas psicológicos para pacientes con síntomas persistentes. Pese a estos lineamientos, existe una baja eficiencia en su manejo ya que desgraciadamente estas guías están basadas principalmente en tratamientos, sin incorporar la prevención, conteniendo 
además datos limitados de su costo efectividad (Foster et al., 2018). Un diseño inclusivo de terapias para el lumbago ayuda a identificar dificultades que podrían afectar el acceso de los pacientes a los servicios (Liu et al., 2019).

El objetivo de este artículo de revisión es presentar una mirada actualizada del lumbago en entornos laborales dada su alta incidencia, permitiendo comprender qué es, sus causas, factores de riesgo, manejo clínico e intervenciones ergonómicas

\section{Materiales y métodos}

Mediante una revisión sistemática cualitativa (Aguilera, 2014) en bases de datos como ScienceDirect, PubMed y Scinapse, con criterios de inclusión: publicaciones en revistas de ergonomía y salud, antigüedad inferior a 10 años (salvo excepciones de alta calidad), se seleccionaron artículos con las palabras clave "lumbago y ergonomía".

\section{Resultados y discusión}

\section{Contexto histórico}

La anatomía evolucionó desde los cuadrúpedos a la bipedestación. Esta nueva postura se asocia a una limitación: mantener un adecuado tono muscular para equilibrar esfuerzos y estructuras osteoarticulares. Este proto-humano estaba diseñado para una vida muy activa y corta (30 años). Con el desarrollo de la agricultura y la revolución industrial, desaparecieron los depredadores y se acumularon recursos, siendo necesario almacenarlos, trans- portarlos y manipularlos. Surgieron dietas hipercalóricas de fácil digestión, favoreciendo la obesidad, perdiendo tono muscular y masa ósea, realizando sobreesfuerzos que llevaron las articulaciones a posturas incorrectas, facilitando su degeneración, surgiendo una epidemia terrible, el lumbago (Vidal, 2017).

A los trastornos musculoesqueléticos se les reconoce factores etiológicos ocupacionales desde el siglo XVIII y pese a que se han publicado miles de artículos científicos, todavía su relación con el trabajo es sujeto de debate (Vranceanu et al., 2009; Bruce, 1997).

\section{Conceptos básicos}

Las vértebras están unidas entre sí por un disco de fibrocartílago que cuenta con un área central elástica rodeada por un anillo fibroso, conocido como disco intervertebral, que funciona absorbiendo impactos. Los discos constituyen el $25 \%$ del largo total de la columna vertebral; su degeneración natural comienza en la vida adulta produciendo una disminución de la estatura. La compresión de los discos durante el día puede causar la pérdida de hasta 1,3 centímetros de estatura. Cada par de vértebras unidas por músculos y ligamentos conforman un pequeño brazo de palanca, sin embargo, la resultante final de toda la columna es de menor potencia que la generada por los miembros (Murrell, 1965). 


\section{¿Qué es el lumbago?}

Es un síntoma y no una enfermedad, pudiendo tener distintos orígenes, definiéndose como un dolor situado entre el borde inferior de las costillas y por encima del pliegue inferior de los glúteos (Hartvigsen \& Hancock, 2018; Dionne et al., 2008). En la mayoría de los pacientes no es posible localizar la fuente nociceptiva, catalogándose en estos casos como lumbago inespecífico (Maher et al., 2017) y solo una pequeña proporción requiere diagnóstico y manejo especializado. Los portadores de lumbago a menudo sufren más dolores y más problemas de salud física y/o mental que la población general (Vranceanu et al., 2009). El lumbago agudo dura menos de 6 semanas, el crónico más de 3 meses y para ambos el mecanismo del dolor es pobremente entendido (Brisby, 2016).

¿Qué puede doler?

\section{Disco intervertebral}

Su daño es la fuente fundamental de dolor en el lumbago crónico (Brisby, 2016).

\section{Facetas articulares}

Están altamente inervadas y responden ante la inflamación con generación de estímulos nociceptivos (Brisby, 2016). La inyección de anestésicos locales en las articulaciones facetarias alivia el dolor, demostrando la participación de ellas en la génesis del lumbago (Maas et al., 2015).

\section{Placas vertebrales terminales}

Nociceptores presentes en los cuerpos vertebrales son estimulados principalmente por fracturas compresivas en presencia de osteoporosis, secundarias a traumas pequeños, flexión de columna o levantamiento de cargas (Brisby, 2016). La respuesta proinflamatoria causada por el daño estructural de la placa terminal o del disco permitiría infiltración microbiana y/o reacciones autoinmunes que incrementan la reacción nociceptiva (Dudli et al., 2016).

\section{Ligamentos}

El longitudinal posterior tiene terminales nerviosas con nociceptores estimulables tanto por compresión como por mediadores inflamatorios (Brisby, 2016).

\section{Raíces nerviosas}

El dolor radicular ocurre por compresión de nervios espinales. La herniación de un disco con inflamación local es su causa más común (Brinjikji, 2015) y si está asociada a lumbago tiene peor pronóstico (Kongsted et al., 2013).

Si bien las causas del lumbago no son identificables en la mayoría de los casos, se postula que la distensión o el espasmo muscular es el origen más probable, desconociéndose si es un fenómeno primario o reactivo (Brisby, 2016; Bruce 1997). El lumbago también puede ser producido por grados moderados de malas posturas sostenidas en el tiempo, por daño muscular, ligamentoso o lesión del complejo discal (Murrell, 1965). 


\section{Causas específicas de lumbago}

En países desarrollados, patologías específicas responsables de lumbago agudo corresponden al $0,9 \%$ de los casos y en los países pobres al 9,5\%, destacando fracturas vertebrales, enfermedades inflamatorias, neoplasias, infecciones, causas intraabdominales y el síndrome de "cauda equina" (Hartvigsen \& Hancock, 2018; Henschke et al., 2009; Galukande et al., 2005). Debemos sospecharlos cuando existan las alertas llamadas "banderas rojas", que en la población trabajadora incluyen trauma, cólicos renales, infecciones urinarias, cáncer, inmunosupresión y déficits neurológicos. La ausencia de estas "alertas" generalmente descarta la necesidad de exámenes, derivaciones a centros de mayor complejidad u hospitalizaciones (Hegmann et al., 2019).

\section{Factores de riesgo}

El lumbago es de origen multifactorial (figura 3) y puede tener relación con condiciones ocupacionales y/o comunes (Andini, 2015; Kerr et al., 2001).

Figura 3. Contribuyentes al lumbago e incapacidad.

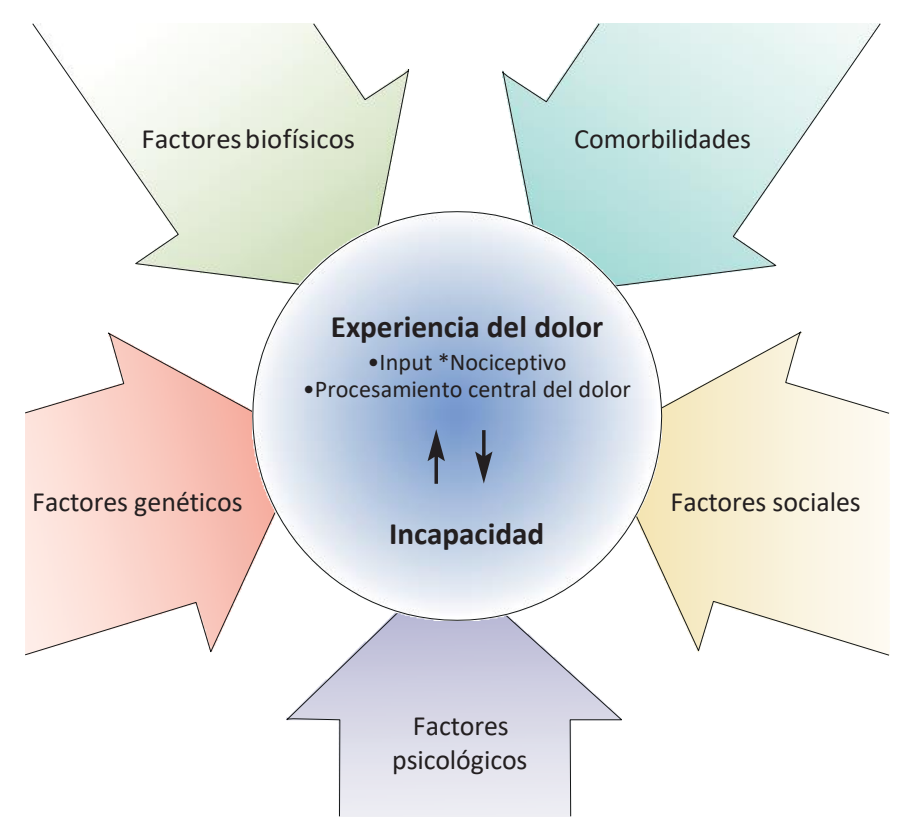

Nota. El modelo incluye contribuyentes claves al lumbago e incapacidad, pero no intenta representar las complejas interacciones entre los diferentes contribuyentes. ${ }^{*}$ Inputs Nociceptivos incluyen fuentes no identificables de dolor en lumbago inespecífico, fuentes neurológicas (p.e., dolor radicular) y patologías específicas (p.e. fracturas). Fuente. Adaptado de Low Back Pain 1 (Lancet, 2018).

\section{Factores biofísicos}

Hay evidencia que el lumbago se asocia a factores ocupacionales de origen biofísico, variando su incidencia en las diferentes industrias (Manchikanti et al., 2014; Kerr et al., 2001; Bruce,1997): 


\section{Trabajo físico pesado}

Es aquel trabajo que impone altas demandas de energía y genera grandes fuerzas compresivas sobre la columna (Nimbarte et al., 2013; Marras et al., 1995). Empujar y tirar objetos con ruedas representa parte relevante de las actividades modernas de manipulación en las industrias, demostrándose correlación entre estas acciones y la ocurrencia de lumbago (Botti et al., 2020). Tirar induce mayores cargas compresivas y empujar desencadena mayores fuerzas de cizallamiento sobre la columna lumbar (Nimbarte et al., 2013; Knapik \& Marras, 2009). La degeneración discal ocurre más temprano en la vida de personas que realizaban trabajos pesados. La severidad del lumbago está relacionada con lo pesado del trabajo (Schroeder et al., 2015; Videman et al., 1990).

\section{Levantamiento de cargas}

La columna se ve afectada por sobrecarga durante levantamientos por la combi- nación de las siguientes variables: Frecuencia de levantamiento, Momento de carga, Velocidad lateral del tronco y Ángulo sagital. Existe fuerte asociación entre levantamiento, torsión del tronco, posturas forzosas y desórdenes lumbares (Coenen et al., 2014; Bruce, 1997; Marras et al., 1995).

\section{Flexión y torsión}

La inclinación lateral o anterior del tronco, asociado a cuclillas, agacharse o arrodillarse, mostró un riesgo elevado de trastornos de columna lumbar con fuerte asociación entre estas posturas forzadas y lumbago (Coenen et al., 2014; Bruce, 1997). La flexión genera fuerzas compresivas y la torsión rápida fuerzas rotacionales o de cizalla en la columna lumbar (Coenen et al., 2014; Griffith et al., 2012; Marras et al., 1995).

\section{Vibraciones}

Hay fuerte asociación entre exposición a vibraciones de cuerpo completo y tras- tornos lumbares. Este factor puede causar enfermedad de la columna por daño mecánico o cambios en el metabolismo de los tejidos, afectando el funciona- miento de músculos, disminuyendo su respuesta y deteriorando la estabilidad de estructuras adyacentes (Burström et al., 2015; Wikström et al., 1994).

\section{Posturas estáticas}

Son posiciones isométricas o inactivas, donde ocurren pequeños movimientos, que causan carga sobre músculos, incluyendo largos períodos sentado, de pie y trabajo sedentario. Existe una alta proporción de patología de discos lumbares en gente con historias de sedentarismo. El trabajo sedentario contribuye al desarrollo de hallazgos patológicos en la columna (Bontrup, 2019; Skov, 1996; Videman et al., 1990). El estado sedente prolongado parece ser un factor de riesgo para lumbago (Villar, 2015; Riihimäki, 1991).

Estos factores ocupacionales pueden ser responsables del desarrollo de cambios patológicos y del incremento de los episodios de lumbago e incapacidad (Schroeder, 2015; Videman et al., 1990). 


\section{Comorbilidades}

Individuos con patologías crónicas como asma, cefalea y diabetes tienen más predisposición a sufrir lumbago que la población general (Parreira et al., 2018). Otras condiciones como el consumo de alcohol (Haiou \& Haldeman, 2016), tabaquismo (Manchikanti et al., 2014; Shiri et al., 2010), mal estado físico (Heneweer et al., 2012; Verbunt et al., 2010), obesidad (Corbeil et al., 2019; Zhang et al., 2018), dislipidemia (Durán-Nah et al., 2016) y anormalidades estructurales se correlacionan con mayor incidencia de lumbago.

\section{Factores sociales}

El lumbago afecta significativamente más a individuos con bajo nivel socio eco- nómico y escolar, lo cual se explica por exposiciones ambientales y estilos de vida deletéreos para su salud, además de una baja accesibilidad a servicios sanitarios. Los factores de riesgo psicosociales, como percepciones de carga de trabajo aumentada, funciones monótonas, bajo control sobre tareas, poca claridad de tarea y bajo apoyo social, se asocian a trastornos musculoesqueléticos. Pese a que sus mecanismos etiológicos son pobremente entendidos, existe evidencia de su rol en el desarrollo de trastornos de columna lumbar (Yang et al., 2016; Vranceanu et al., 2009; Bruce, 1997; Garg \& Moore, 1992).

\section{Factores psicológicos}

La presencia de depresión, ansiedad, pesimismo y percepción de baja autoeficacia en individuos que sufren lumbago se asocia a una mayor incidencia de in- capacidad por esta condición. La percepción alterada de la magnitud del dolor juega un rol crítico en su evolución, afectando la experiencia del dolor, pudiendo favorecer un lumbago crónico, caracterizado por mal funcionamiento cortical, dolor difuso no sistematizable y patrones de sueño alterados; estos individuos experimentan una mentalidad, actitud y creencias negativas respecto al dolor que incrementan su incapacidad e interfieren actividades de la vida diaria (Adnan et al., 2020).

\section{Factores genéticos}

Se ha demostrado influencia genética en el desarrollo de lumbago entre el 21\% y el $67 \%$ de casos relacionados con cuadros crónicos e incapacitantes, corroborado en estudios genéticos y epidemiológicos con gemelos, quienes presentan similares incidencias de afecciones lumbares (Urban \& Fairbank, 2020; Manchikanti et al., 2014).

\section{Factores protectores}

Escolaridad secundaria o superior, ausencia de dislipidemia, ejercicio físico y no manipular objetos pesados se asociaron con menor riesgo de lumbago (Durán-Nah et al., 2016; Kwon et al., 2006).

\section{Diagnóstico}

Existen pruebas diagnósticas anatómicas, funcionales, fisiológicas y biomarcadores. Las Anatómicas corresponden a imágenes que ayudan a definir estructuras, obteniéndose por medio de radiografías, resonancia magnética, tomografía computada, mielogramas y pruebas de medicina nuclear. Las funcionales incluyen pruebas clínicas que evalúan la capacidad del 
paciente para movilizar una extremidad. Las fisiológicas consideran las electromiografías de superficie y las con aguja (Hegmann et al., 2019).

\section{Biomarcadores}

\section{Encefálicos}

El lumbago está asociado con cambios estructurales y funcionales generalizados en el cerebro que se correlacionan con este dolor y sus respuestas emocionales. La resonancia magnética estructural de encéfalo muestra aumento del grosor cortical en áreas específicas; también se detectan cambios en la resonancia funcional: la conectividad funcional en estado de reposo de las redes apoya los hallazgos de alteraciones de la estructura y funciones cerebrales localizadas en regiones correspondientes a funciones cognitivas, dimensiones viso motoras y afectivas de procesamiento del dolor. Estas modificaciones son biomarcadores que permitirán guiar la terapia (Lamichhane et al., 2021).

\section{Humorales}

Diferentes sustancias inflamatorias, señalizadoras y moduladoras como el factor de necrosis tumoral, interleukinas y óxido nítrico se han detectado en hernias discales (Brisby, 2016). La interleukina 6 y la creatinkinasa se han medido en in- dividuos sometidos a cargas, flexiones y extensiones lumbares, mostrando un ascenso significativo asociado a dosis respuesta. Esta alza está relacionada con daño muscular y apoya evidencia epidemiológica previa que puede tener valor para predecir riesgo de injuria ocupacional (Christian \& Nussbaum, 2015).

Los exámenes diagnósticos no están indicados en pacientes con lumbago inespecífico

No existe evidencia que imágenes obtenidas por resonancia mejoren el pronóstico de los pacientes (Steffens et al., 2014). Las hernias discales son hallazgos frecuentes en resonancias de población general y a menudo desaparecen independiente de la resolución del dolor (Brisby, 2016; Chiu et al., 2015). Las guías clínicas no recomiendan usar rutinariamente esta herramienta en pacientes con lumbago inespecífico (Qaseem et al., 2017; Wong et al., 2017). Las imágenes incrementan la medicalización y realización innecesaria de exámenes y tratamientos, solo logrando retrasar la recuperación, teniendo el potencial de incrementar significativamente los efectos adversos. Pacientes con banderas rojas son la "excepción a la regla” que señala: “evitar exámenes en la mayoría de los pacientes”(Hegmann et al., 2019).

\section{Tratamiento}

Pese a las recomendaciones en guías clínicas, existen importantes diferencias entre la evidencia formal y la práctica (Foster et al., 2018). Actualmente guías de países desarrollados privilegian el automanejo, terapias físicas, psicológicas, medicina complementaria y no los tratamientos farmacológicos o quirúrgicos como primera alternativa, reservándolos para quienes el manejo inicial sin medicamentos no funcionó (Qaseem et al., 2017). 
Tabla 1. Intervenciones aprobadas para manejo del lumbago inespecífico por las Guías de Dinamarca, EE. UU. y Reino Unido.

\begin{tabular}{|c|c|c|}
\hline $\begin{array}{l}\text { Educación y } \\
\text { autocuidado }\end{array}$ & $\begin{array}{c}\text { Lumbago agudo } \\
\text { (<6 semanas) }\end{array}$ & $\begin{array}{c}\text { Lumbago persistente } \\
\text { (<12 semanas) }\end{array}$ \\
\hline Mantenerse activo & \multicolumn{2}{|l|}{ Tratamiento de primera línea } \\
\hline Educación & \multicolumn{2}{|l|}{ Tratamiento de primera línea } \\
\hline Calor superficial & \multicolumn{2}{|l|}{ Segunda línea o coadyuvante } \\
\hline \multicolumn{3}{|c|}{ Terapia no farmacológica } \\
\hline \multicolumn{3}{|c|}{ Terapia de ejercicios } \\
\hline \multicolumn{3}{|c|}{ Uso limitado en pacientes seleccionados } \\
\hline \multicolumn{3}{|c|}{ Tratamiento primera línea } \\
\hline \multicolumn{3}{|l|}{ Considere uso de rutina } \\
\hline \multicolumn{3}{|c|}{ Terapia cognitiva conductual } \\
\hline \multicolumn{3}{|c|}{ Uso limitado en pacientes seleccionados } \\
\hline \multicolumn{3}{|c|}{ Tratamiento primera línea } \\
\hline \multicolumn{3}{|c|}{ Considere uso de rutina } \\
\hline \multicolumn{3}{|l|}{ Manipulación espinal } \\
\hline \multicolumn{3}{|c|}{ Segunda línea o terapia coadyuvante } \\
\hline \multicolumn{3}{|c|}{ Masaje } \\
\hline \multicolumn{3}{|c|}{ Segunda línea o terapia coadyuvante } \\
\hline \multicolumn{3}{|l|}{ Acupuntura } \\
\hline \multicolumn{3}{|c|}{ Segunda línea o terapia coadyuvante } \\
\hline \multicolumn{3}{|c|}{ Yoga } \\
\hline \multicolumn{3}{|l|}{ Evidencia insuficiente } \\
\hline \multicolumn{3}{|c|}{ Segunda línea o terapia coadyuvante } \\
\hline \multicolumn{3}{|c|}{ Reducción del estrés } \\
\hline \multicolumn{3}{|l|}{ Evidencia insuficiente } \\
\hline \multicolumn{3}{|c|}{ Segunda línea o terapia coadyuvante basada en Mindfulness } \\
\hline \multicolumn{3}{|l|}{ Rehabilitación } \\
\hline \multicolumn{3}{|l|}{ Evidencia insuficiente } \\
\hline Segunda línea o terapi & anterdisciplinaria coadyuvante & \\
\hline Terapia farmacoló & ica & \\
\hline Paracetamol & & \\
\hline No recomendado & & \\
\hline Antinflamatorios no $\mathrm{e}$ & steroidales & \\
\hline Segunda línea o terap & a coadyuvante & \\
\hline Relajantes musculare & & \\
\hline Evidencia insuficiente & & \\
\hline Uso limitado en pacie & tes seleccionados & \\
\hline Inhibidores recaptaci & in norepinefrina & \\
\hline Evidencia insuficiente & & \\
\hline Segunda línea o terap & a coadyuvante & \\
\hline Anticonvulcionantes & & \\
\hline
\end{tabular}




\begin{tabular}{|l|}
\hline Evidencia insuficiente \\
Segunda línea o terapia coadyuvante \\
Opiáceos \\
Uso limitado en pacientes seleccionados \\
Rol incierto \\
Glucocorticoides sistémicos \\
No recomendado \\
Uso limitado en pacientes seleccionados \\
\hline Terapias intervencionales \\
\hline Inyección epidural con glucocorticoides (para disco herniado con radiculopatía) \\
No recomendado \\
Uso limitado en pacientes seleccionados \\
\hline Cirugía \\
\hline Discectomía (para disco herniado con radiculopatía) \\
Evidencia insuficiente \\
Segunda línea o terapia coadyuvante \\
Laminectomía (para estenosis espinal sintomática) \\
Evidencia insuficiente en estenosis espinal \\
Segunda línea o terapia coadyuvante \\
Fusión espinal (para lumbago no radicular con hallazgos discales) \\
Evidencia insuficiente \\
Rol incierto
\end{tabular}

Nota. El lumbago subagudo es un período de transición entre el agudo y el crónico; evidencia para el manejo del lumbago subagudo es- casa, pero una aproximación razonable es cambiar hacia las terapias recomendadas para el lumbago crónico. Fuente. Adaptado de Guías de Manejo (Foster et al., 2018).

Existe poca investigación en prevención, sólo demostrándose la utilidad de los ejercicios y la educación; la evidencia de la efectividad de la prevención primaria para lumbago es inadecuada (Hartvigsen \& Hancock, 2018).

Tabla 2. Evidencia de estrategias de prevención para lumbago: Conclusiones de efectividad y grados de fuerza o rango de eficacia. Tomado de revisiones sistemáticas.

\begin{tabular}{|l|l|}
\hline \multicolumn{2}{|c|}{ Efectos y calidad de la evidencia } \\
\hline Ejercicios y educación & Efectivo (calidad moderada) \\
\hline Ejercicio & Efectivo (baja calidad) \\
\hline Educación & Inefectivo (calidad moderada) \\
\hline Back belt & Inefectivo (calidad muy baja) \\
\hline Plantillas de zapatos & Inefectivo (baja calidad) \\
\hline Intervenciones ergonómicas en el lugar de trabajo & Inefectivo (calidad moderada) \\
\hline
\end{tabular}

Fuente. Adaptado de Low Back Pain 2 (Lancet 2018).

Tratamiento médico (Qaseem et al., 2017)

Como la mayoría de los pacientes con lumbago agudo mejoran independiente del tratamiento, debe elegirse el calor superficial, masaje, acupuntura o manipulaciones de columna. Si se desea administrar fármacos, debe elegirse antiinflamatorios no esteroidales o 
relajantes musculares (recomendación fuerte).

En lumbago crónico, inicialmente elegir tratamiento no farmacológico que incluya ejercicios, rehabilitación, acupuntura, reducción del stress, Thai Chi, Yoga, relajación progresiva y terapia cognitivo conductual (recomendación fuerte).

En lumbago crónico con respuesta inadecuada a terapia no farmacológica, considerar antiinflamatorios no esteroidales, tramadol o duloxetina como segunda línea (recomendación débil).

\section{Fisioterapia}

Los ejercicios para el lumbago son útiles, describiéndose más de 33 mecanismos diferentes de acción, siendo los neuromusculares y psicosociales los más relevantes, sin embargo, no hay consenso del por qué funcionan (Wun et al., 2021; Gordon \& Bloxham, 2016).

Ejercicios de estabilización del Core aumentaron el grosor de los músculos trans- verso abdominal y glúteo mayor, disminuyendo la incapacidad en individuos con lumbago inespecífico (Narouei et al., 2020).

\section{Ergonomía}

Un enfoque anticipativo exitoso debe involucrar el estudio de capacidades y características humanas que intervienen en la concepción de equipamientos, sistemas y actividades, cuyos objetivos sean la mejora continua, eficiencia, seguridad y bienestar de los individuos (Fernandes et al., 2015; Clark, 1991). El ergónomo que trabaja en forma aislada no tendrá éxito en vencer los trastornos musculoesqueléticos, por lo cual será fundamental contar con el involucra- miento gerencial para lograr procesos racionalizados en forma balanceada que impacten la productividad y bienestar de los trabajadores (Rodríguez et al., 2012; Winkel \& Westgaard, 1996).

Aspectos ergonómicos para considerar en la prevención del lumbago:

1. No se debe solicitar a los operadores ejecutar fuerzas en posturas que los obligue a doblar su espalda (Murrell, 1965).

2. Conocer el mecanismo de funcionamiento de la columna vertebral es de importancia cuando se diseñan equipos que involucren levantamiento de cualquier naturaleza, o donde se aplique fuerza con los brazos (Murrell, 1965).

3. Respetar las Normas: La ISO 11228-2 (2007) y el Informe Técnico ISO (TR) 12295 (2014) describen la metodología para identificar condiciones aceptables al empujar y tirar en el trabajo, proporcionando instrucciones para el rediseño ergonómico de estas tareas, basado en los estudios de Snook y Ciriello, quienes emplearon un enfoque psicofísico para determinar las fuerzas máximas aceptables de empuje y tracción. La evidencia actual demuestra que este requisito no se respeta en muchas industrias, favoreciendo la aparición de dolor lumbar (Botti et al., 2020; Snook \& Ciriello 1991).

4. Cargas equivalentes al 20\% del peso corporal son el límite de esfuerzos aceptables al tirar. Estos hallazgos proporcionan información del riesgo potencial para la zona lumbar durante esfuerzos (Diego-Mas, 2015; Knapik \& Marras, 2009). 
5. En tareas de manipulación, la restricción de la movilidad de la cadera por pan- talones ajustados produce una respuesta compensatoria de mayor movimiento lumbar y niveles reducidos de activación de los músculos del tronco, constituyendo un posible factor en el aumento de tensión en estructuras lumbares, pudiendo ser causa potencial de lumbago (Eungpinichpong et al., 2013).

6. El efecto acumulativo de múltiples repeticiones en aceleraciones de carros en combinación con agacharse y levantarse, habitualmente de forma incómoda, puede ser causa de lumbago en azafatas. Las recomendaciones para auxiliares de cabina es evitar tirar el carro, reducir su rapidez especialmente al girar y solo re- alizar aceleraciones repetitivas con inclinación negativa del piso (Sandfeld et al., 2014).

7. Exoesqueletos reducen las fuerzas musculares de la región lumbar, disminuyendo las molestias en la zona (Bosch et al., 2016).

8. Existe evidencia que un sistema de levantamiento por vacío disminuyó en un $39 \%$ las fuerzas de compresión lumbar, bajo el límite de daño (3400 N). También redujo en 25\% las fuerzas de cizalla anteroposteriores en L5-S1 (Lu et al., 2018).

9. La experiencia influye en variables relacionadas con la postura al levantar cargas. Trabajadores expertos flectan menos su columna lumbar y más sus rodillas, mejorando la distribución de las fuerzas internas en la columna; sin embargo, produce pequeños efectos en las variables de carga externa. Una intervención ergonómica debe tener como foco primario reducir las cargas externas de columna más que sofisticar la técnica del trabajador (Plamondon et al., 2014).

10. Muchos productos mineros se empacan en bolsas pequeñas cargadas en pallets, requiriendo manejo manual el cual se asocia a lumbago. En muchos casos se excede el criterio NIOSH de $3400 \mathrm{~N}$. La orientación del pallet tiene impacto significativo en la compresión de columna lumbar; posicionándolo correctamente se reducen las cargas en 800 N (Gallagher \& Heberger, 2015).

\section{Conclusiones}

El lumbago es un síntoma común a nivel global en todo grupo etario, siendo la principal causa de incapacidad, impactando más en países de ingresos bajos y medios, donde es limitada la posibilidad de modificar puestos de trabajo. La mayoría son episodios autolimitados con recurrencias frecuentes. Su origen es multifactorial, siendo complejo identificar la fuente de dolor. Sus costos son importantes, proyectándose un continuo ascenso. Las Guías Clínicas recomiendan automanejo, terapias físicas, psíquicas y complementarias, pero la realidad demuestra que el uso liberal de imágenes, otros exámenes, medicamentos y cirugías es una práctica común que deteriora su pronóstico. Existe poca investigación en prevención, sólo demostrándose la utilidad de los ejercicios y la educación. Soluciones prometedoras son la implementación de las mejores prácticas clínicas, integrar los sistemas sanitarios al cuidado ocupacional e incorporar la ergonomía de forma anticipativa, sistémica y participativa. 


\section{Referencias}

Adnan, R., Van Oosterwijck, J., Danneels, L., Willems, T., Meeus, M., Crombez, G., \& Goubert, D., (2020). Differences in psychological factors, disability and fatigue according to the grade of chronification in non-specific low back pain patients: A cross - sectional study. Journal of Back and Musculoskeletal Rehabilitation, 33(6), 919-930. https://doi.org/10.3233/BMR$\underline{191548}$

Andini, F. (2015). Risk factors of low back pain in workers. Medical Journal of Lampung University, 4(1), 12-19. https://juke.kedokteran.unila.ac.id/index.php/majority/article/view/495

Anon. (2013). Liberty Mutual Workplace Safety Index.

Lardon, A., Dubois, J. D., Cantin, V., Piché, M., \& Descarreaux, M. (2018). Predictors of disability and absenteeism in workers with non-specific low back pain: A longitudinal 15-month study. Applied Ergonomics, 68, 176-185. https://doi.org/10.1016/j.apergo.2017.11.011

Blach, C. R., Høybyeab, M. T., Bastrup, L. J., Deigaard, L. B., \& Hybholt, L. (2021). Disrupted everyday life in the trajectory of low back pain: A longitudinal qualitative study of the crosssectorial pathways of individuals with low back pain over time. International Journal of Nursing Studies Advances, 3, 100021. https://doi.org/10.1016/J.IJNSA.2021.100021

Bontrup, C., Taylor, W. R., Fliesser, M., Visscher, R., Green, T., Wippert, P. M., \& Zemp, R. (2019). Low back pain and its relationship with sitting behaviour among sedentary office workers. Applied ergonomics, 81, 102894. https://doi.org/10.1016/j.aperg0.2019.102894

Bosch, T., Van Eck, J., Knitel, K., \& De Looze, M. (2016). The effects of a passive exoskeleton on muscle activity, discomfort and endurance time in forward bending work. Applied Ergonomics, 54, 212-217. https://doi.org/10.1016/j.apergo.2015.12.003

Botti, L., Galizia, F., \& Zecchi, G. (2020). A thorough investigation on pushing activities in industry: The impact of the variation in the speed of motion and load conditions on initial and sustained forces. Applied Ergonomics, 85, 103080. https://doi.org/10.1016/j.apergo.2020.103080

Brinjikji, W., Luetmer, P. H., Comstock, B., Bresnahan, B. W., Chen, L. E., Deyo, R. A., Halabi, S., Turner, J. A., Avins, A. L., James, K., Wald, J. T., Kallmes, D. F., \& Jarvik, J. G. (2015). Systematic literature review of imaging features of spinal degeneration in asymptomatic populations. American journal of neuroradiology, 36(4), 811-816. https://doi.org/10.3174/ajnr.A4173

Brisby, H. (2016). Pain origin and mechanisms in low back pain. En E. Van de Kelft (ed.), Surgery of the spine and spinal cord. Springer. https://doi.org/10.1007/978-3-319-27613-7 24

Bruce, P. (1997). Musculoskeletal disorders and workplace factors. A critical review of epidemiologic evidence for work-related musculoskeletal disorders of the neck upper extremity, and low back. Public Health Service for Disease Control and Prevention National Institute for Occupational Safety and Health.

Burström, L., Nilsson, T., \& Wahlström, J. (2015). Whole-body vibration and the risk of low back pain and sciatica: a systematic review and meta-analysis. International Archives of Occupational and Environmental Health, 88, 403-418. https://doi.org/10.1007/s00420014-0971-4

Cassidy, J. D., Carroll, L. J., \& Côté, P. (1998). The Saskatchewan health and back pain survey. The prevalence of low back pain and related disability in Saskatchewan adults. Spine, 23(17), 1860-1867. https://doi.org/10.1097/00007632-199809010-00012 
Chiu, C. C., Chuang, T. Y., Chang, K. H., Wu, C. H., Lin, P. W., \& Hsu, W. Y. (2015). The probability of spontaneous regression of lumbar herniated disc: a systematic review. Clinical rehabilitation, 29(2), 184-195. https://doi.org/10.1177/0269215514540919

Christian, M., \& Nussbaum M. A. (2015). Responsiveness of selected biomarkers of tissue damage to external load and frequency during repetitive lumbar flexion/ex- tension. International Journal of Industrial Ergonomics, 48, 1-9. https://doi.org/10.1016/j.ergon.2015.03.001

Coenen, P., Gouttebarge, V., Van der Burght, A., Van Dieën, J. H., Frings-Dresen, M., Van der Beek, A., \& Burdorf, A. (2014). The effect of lifting during work on low back pain: A health impact assessment based on a meta-analysis. Occupational and Environmental Medicine, 71, 871-877. http://doi.org/10.1136/oemed-2014-102346

Coombs, D. M., Machado, G. C., Richards, B., Wilson, R., Chan, J., Storey, H., Maher, G. C. (2021). Healthcare costs due to low back pain in the emergency department and in patient setting in Sydney Australia. The Lancet Regional Health - Western Pacific, 7, 100089. https://doi.org/10.1016/j.lanwpc.2020.100089

Corbeil, P., Plamondon, A., Handrigan, G., Vallée-Marcotte, J., Laurendeau, S., Ten Have, J., \& Manzerolle, N. (2019). Biomechanical analysis of manual material handling movement in healthy weight and obese workers. Applied ergonomics, 74, 124-133. https://doi.org/10.1016/j.apergo.2018.08.018

Da Silva, T., Mills, K., Brown, B. T., Pocovi, N., de Campos, T., Maher, C., \& Hancock, M. J. (2019). Recurrence of low back pain is common: A prospective inception cohort study. Journal of physiotherapy, 65(3), 159-165. https://doi.org/10.1016/j.jphys.2019.04.010

Deyo, R. A., \& Tsui-Wu, Y. J. (1987). Descriptive epidemiology of low-back pain and its related medical care in the United States. Spine, 12(3), 264-268. https://doi.org/10.1097/00007632-198704000-00013

Diego-Mas, J. A. (2015). Evaluación de la manipulación manual de cargas mediante las tablas de Snook y Ciriello. Ergonautas.

Dionne, C. E., Dunn, K. M., Croft, P. R., Nachemson, A. L., Buchbinder, R., Walker, B. F., Wyatt, M., Cassidy, J. D., Rossignol, M., Leboeuf-Yde, C., Hartvigsen, J., Leino-Arjas, P., Latza, U., Reis, S., Gil Del Real, M. T., Kovacs, F. M., Oberg, B., Cedraschi, C., Bouter, L. M., Koes, B. W., ... Von Korff, M. (2008). A consensus approach toward the standardization of back pain definitions for use in prevalence studies. Spine, 33(1), 95-103. https://doi.org/10.1097/BRS.obo13e31815e7f94

Dudli, S., Fields, A. J., Samartzis, D., Karppinen, J., \& Lotz, J. C. (2016). Pathobiology of modic changes. European spine journal: Official publication of the European Spine Society, the European Spinal Deformity Society, and the European Section of the Cervical Spine Research Society, 25(11), 3723-3734. https://doi.org/10.1007/s00586-016-4459-7

Durán-Nah, J. J., Benítez-Rodríguez, C. R., \& Miam-Viana, E. J. (2016). Lumbalgia crónica y factores de riesgo asociados en derechohabientes del IMSS: Estudio de casos y controles. Revista Médica del Instituto Mexicano del Seguro Social, 54(4), 421-428. https://www.medigraphic.com/cgi-bin/new/resumen.cgi?IDARTICULO=66911

Foster, N. E., Anema, J. R., Cherkin, D., Chou, R., Cohen, S. P., Gross, D. P., Ferreira, P. H., Fritz, J. M., Koes, B. W., Peul, W., Turner, J. A., Maher, C. G., \& Lancet Low Back Pain Series Working Group. (2018). Prevention and treatment of low back pain: Evidence, challenges, and promising directions. Lancet, 391(10137), 2368-2383. https://doi.org/10.1016/So1406736(18)30489-6

Freburger, J,. K., Holmes, G. M., Agans, R. P., Jackman A. M., Darter, J. D., Wallace, A. S., Castel, L. D., Kalsbeek, W. D., \& Carey, T. S. (2009). The rising prevalence of chronic low back pain. 
$\begin{array}{llll}\text { Archives of internal } & \text { 251-8. }\end{array}$ https://doi.org/10.1001/archinternmed.2008.543

Gallagher, S., \& Heberger, J. R. (2015). The effects of operator position, pallet orientation, and palletizing condition on low back loads in manual bag palletizing operations. International Journal of Industrial Ergonomics, 47, 84-92. https://doi.org/10.1016/j.ergon.2015.03.005

Galukande, M., Muwazi, S., \& Mugisa, D. B. (2005). Etiology of low back pain in Mulago Hospital, Uganda. African Health Sci, 5, 164-167.

Garg, A., \& Moore, S. J. (1992). Epidemiology of low-back pain in industry. Occupational Medicine: State of the Art Reviews, 7(4), 593-608.

Global Burden of Disease. (2015). Disease and injury incidence and prevalence collaborators. Global, regional, and national incidence, prevalence, and years lived with disability for 310 diseases and injuries, 1990-2015: A systematic analysis for the Global Burden of Disease Study 2015. Lancet, 388(10053), 1545-1602. https://doi.org/10.1016/So140-6736(16)31678$\underline{6}$

Global Health Data Exchange. (2021). Institute for health metrics and evaluation. University of Washington.

Griffith, L. E., Shannon, H. S., Wells, R. P., Walter, S. D., Cole, D. C., Côté, P., Frank, J., HoggJohnson, S., \& Langlois, L. E. (2012). Individual participant data meta-analysis of mechanical workplace risk factors and low back pain. American journal of public health, 102(2), 309-318. https://doi.org/10.2105/AJPH.2011.300343

Gordon, R., \& Bloxham, S. (2016). A systematic review of the effects of exercise and physical activity on non-specific chronic low back pain. Healthcare, 4, 22.

Guo, H. R., Tanaka, S. H., \& Alperin, W. E. (1999). Low back pain prevalence in US industry and estimates of lost workdays. American Journal of Public Health, 89(7) 1029-1035.

Haiou, Y., \& Haldeman, S. (2016). Behavior-related factors associated with low back pain in the $\begin{array}{llll}\text { us adult } & \text { population. } & \text { 28-34. }\end{array}$ https://doi.org/10.1097/BRS.0000000000001665

Hartvigsen, J., \& Hancock, M. J. (2018). What low back pain is and why we need to pay attention. Lancet, 391, 2356-2367.

Hegmann, K. T., Travis, R., Belcourt, R. M., Donelson, R., Eskay-Auerbach, M., Galper, J., Haldeman, S., Hooper, P. D., Lessenger, J. E., Mayer, T., Mueller, K. L., Murphy, D. R., Tellin, W. G., Thiese, M. S., \& Weiss, M. S. (2019). Diagnostic tests for low back disorders. Journal of occupational and environmental medicine, 61(4), e155-e168. https://doi.org/10.1097/JOM.0000000000001551

Heneweer, H., Picavet, H. S., Staes, F., Kiers, H., \& Vanhees, L. (2012). Physical fitness, rather than self-reported physical activities, is more strongly associated with low back pain: evidence from a working population. European spine journal: Official publication of the European Spine Society, the European Spinal Deformity Society, and the European Section of the Cervical Spine Research Society, 21(7), 1265-1272. https://doi.org/10.1007/so0586011-2097-7

Henschke, N., Maher, C. G., Refshauge, K. M., Herbert, R. D., Cumming, R. G., Bleasel, J., York, J., Das, A., \& McAuley, J. H. (2009). Prevalence of and screening for serious spinal pathology in patients presenting to primary care settings with acute low back pain. Arthritis and rheumatism, 6o(10), 3072-3080. https://doi.org/10.1002/art.24853

Hoy, D., March, L., Brooks, P., Woolf, A., Blyth, F., Vos, T., \& Buchbinder, R. (2010). Measuring the global burden of low back pain. Best practice \& research. Clinical rheumatology, 24(2), 155-165. https://doi.org/10.1016/j.berh.2009.11.002 
Informe Técnico ISO (TR) 12295. (2014). Ergonomics - application document for international standards on manual handling (ISO 11228- 1, ISO 11228-2 \& ISO 11228-3) and evaluation of static working postures (ISO 11226) https://www.iso.org/standard/51309.html. 2014

Katz J. N. (2006). Lumbar disc disorders and low-back pain: socioeconomic factors and consequences. The Journal of bone and joint surgery. American volume, $88 \mathrm{Suppl}$ 2, 21-24. https://doi.org/10.2106/JBJS.E.01273

Kerr, M. S., Frank, J. W., Shannon, H. S., Norman, R. W., Wells, R. P., Neumann, W. P., Bombardier, C., \& Ontario Universities Back Pain Study Group (2001). Biomechanical and psychosocial risk factors for low back pain at work. American journal of public health, 91(7), 1069-1075. https://doi.org/10.2105/ajph.91.7.1069

Knapik, G. G., \& Marras, W. S. (2009). Spine loading at different lumbar levels during pushing and pulling. Ergonomics, 52(1), 60-70. https://doi.org/10.1080/00140130802480828

Kongsted, A., Kent, P., Jensen, T. S., Albert, H., \& Manniche, C. (2013). Prognostic implications of the Quebec Task Force classification of back-related leg pain: An analysis of longitudinal routine clinical data. BMC musculoskeletal disorders, 14, 171. https://doi.org/10.1186/14712474-14-171

Kwon, M. A., Shim, W. S., Kim, M. H., Gwak, M. S., Hahm, T. S., Kim, G. S., Kim, C. S., Choi, Y. H., Park, J. H., Cho, H. S., \& Kim, T. H. (2006). A correlation between low back pain and associated factors: a study involving 772 patients who had undergone general physical examination. Journal of Korean medical science, 21(6), 1086-1091. https://doi.org/10.3346/jkms.2006.21.6.1086

Lamichhane, B., Jayasekera, D., Jakes, R., Glasser, M.F., Zhang, J.K., Yang, C., Grimes, D., Frank, T.L., Ray, W.Z., Leuthardt, E.C., \& Hawasli, A.H. (2021). Multi-modal biomarkers of low back pain: A machine learning approach. NeuroImage: Clinical, 29.

Liu, Y., Dickerson, T., Waddingham, P.E., \& Clarkson, P.J. (2019). Improving people's access to community-based back pain treatment through an inclusive design approach. Applied ergonomics, 81, 102876.

Maas, E. T., Ostelo, R. W., Niemisto, L., Jousimaa, J., Hurri, H., Malmivaara, A., \& van Tulder, M. W. (2015). Radiofrequency denervation for chronic low back pain. The Cochrane database of systematic reviews, (10), CDoo8572. https://doi.org/10.1002/14651858.CDo08572.pub2

Maher, C., Underwood, M., \& Buchbinder, R. (2017). Non-specific low back pain. Lancet, 389(10070), 736-747. https://doi.org/10.1016/S0140-6736(16)30970-9

Manchikanti, L., Singh, V., Falco, F. J., Benyamin, R. M., \& Hirsch, J. A. (2014). Epidemiology of low back pain in adults. Neuromodulation: Journal of the International Neuromodulation Society, 17(Suppl 2), 3-10. https://doi.org/10.1111/ner.12018

Maniadakis, N., \& Gray, A. (2000). The economic burden of back pain in the UK. Pain, 84(1), 95103. https://doi.org/10.1016/S0304-3959(99)00187-6

Marras, W. S., Lavender, S. A., Leurgans, S. E., Fathallah, F. A., Ferguson, S. A., Allread, W. G., \& Rajulu, S. L. (1995). Biomechanical risk factors for occupationally related low back disorders. Ergonomics, 38(2), 377-410. https://doi.org/10.1080/00140139508925111

Lu, M. L., Dufour, J. S., Weston, E. B., \& Marras, W. S. (2018). Effectiveness of a vacuum lifting system in reducing spinal load during airline baggage handling. Applied ergonomics, 7O, 247-252. https://doi.org/10.1016/j.apergo.2018.03.006

Murrell, K. F. H. (1965). Ergonomics. Man in his working environment. John Wiley \& Sons.

The US Burden of Disease Collaborators. (2018). The state of US health, 1990-2016: Burden of diseases, injuries, and risk factors among US States. JAMA, 319(14):1444-1472. https://doi.org/10.1001/jama.2018.0158 
Muslim, K., \& Nussbaum, M. A. (2017). The effects of a simple intervention on exposures to low back pain risk factors during traditional posterior load carriage. Applied ergonomics, 59(Pt A), 313-319. https://doi.org/10.1016/j.aperg0.2016.09.003

Nimbarte, A. D., Sun, Y., Jaridi, M., \& Hsiao, H. (2013). Biomechanical loading of the shoulder complex and lumbosacral joints during dynamic cart pushing task. Applied ergonomics, 44(5), 841-849. https://doi.org/10.1016/j.aperg0.2013.02.008

Norma ISO 11228-2. (2007). Ergonomics manual handling, part 2: Pushing and pulling https://www.iso.org/standard/26521.html

Parreira, P., Maher, C. G., Steffens, D., Hancock, M. J., \& Ferreira, M. L. (2018). Risk factors for low back pain and sciatica: an umbrella review. The spine journal: Official journal of the $\begin{array}{llll}\text { North American Spine } & \text { Society, }\end{array}$ https://doi.org/10.1016/j.spinee.2018.05.018

Plamondon, A., Delisle, A., Bellefeuille, S., Denis, D., Gagnon, D., Larivière, C., \& IRSST MMH Research Group. (2014). Lifting strategies of expert and novice workers during a repetitive palletizing task. Applied ergonomics, 45(3), 471-481. https://doi.org/10.1016/j.apergo.2013.06.008

Qaseem, A., Wilt, T. J., McLean, R. M., Forciea, M. A., Clinical Guidelines Committee of the American College of Physicians, Denberg, T. D., Barry, M. J., Boyd, C., Chow, R. D., Fitterman, N., Harris, R. P., Humphrey, L. L., \& Vijan, S. (2017). Noninvasive treatments for acute, subacute, and chronic low back pain: A clinical practice guideline from the American College of Physicians. Annals of internal medicine, 166(7), 514-530. https://doi.org/10.7326/M16-2367

Riihimäki, H. (1991). Low-back pain, its origin and risk indicators. Scandinavian journal of work, environment \& health, 17(2), 81-90.

Fernandes, P. R., Hurtado, A. L., \& Batiz, E. C. (2015). Ergonomics management with a proactive focus. Procedia Manufacturing, 3, 4509-4516.

Rodríguez, R. Y., Pérez Mergarejo, E., \& Montero, M. R. (2012). Modelo de madurez de ergonomía para empresas (MMEE). El Hombre y La Máquina, (40), 22-30. https://redalyc.org/pdf/478/47826850003.pdf

Sandfeld, J., Rosgaard, C., \& Jensen, B. R. (2014). L4-L5 compression and anterior/posterior joint shear forces in cabin attendants during the initial push/pull actions of airplane meal carts. Applied ergonomics, 45(4), 1067-1075. https://doi.org/10.1016/j.aperg0.2014.01.006

Schroeder, G., Guyre, C. A., Vaccaro, A. R. (2015). The epidemiology and pathophysiology of lumbar disc herniations. Seminars in Spine Surgery, 28(1), 2-7. https://doi.org/10.1053/j.semss.2015.08.003

Narouei, S., Barati, A. H., Akuzawa, H., Talebian, S., Ghiasi, F., Akbari, A., \& Alizadeh, M. H. (2020). Effects of core stabilization exercises on thickness and activity of trunk and hip muscles in subjects with nonspecific chronic low back pain. Journal of Bodywork and Movement Therapies, 24(4), 138-146. https://doi.org/10.1016/j.jbmt.2020.06.026

Shiri, R., Karppinen, J., Leino-Arjas, P., Solovieva, S., \& Viikari-Juntura, E. (2010). The association between smoking and low back pain: A meta-analysis. The American journal of medicine, 123(1). https://doi.org/10.1016/j.amjmed.2009.05.028

Snook, S. H., \& Ciriello, V. M. (1991). The design of manual handling tasks: revised tables of maximum acceptable weights and forces. Ergonomics 34(9), 1197-1213. https://doi.org/10.1080/00140139108964855 
Steffens, D., Hancock, M. J., Maher, C. G., Williams, C., Jensen, T. S., \& Latimer, J. (2014). Does magnetic resonance imaging predict future low back pain? A systematic review. European journal of pain, 18(6), 755-765. https://doi.org/10.1002/j.1532-2149.2013.00427.x

Stochkendahl, M. J., Kjaer, P., Hartvigsen, J., Kongsted, A., Aaboe, J., Andersen, M., Andersen, M. Ø., Fournier, G., Højgaard, B., Jensen, M. B., Jensen, L. D., Karbo, T., Kirkeskov, L., Melbye, M., Morsel-Carlsen, L., Nordsteen, J., Palsson, T. S., Rasti, Z., Silbye, P. F., Steiness, M. Z., ... Vaagholt, M. (2018). National Clinical Guidelines for non-surgical treatment of patients with recent onset low back pain or lumbar radiculopathy. European spine journal: Official publication of the European Spine Society, the European Spinal Deformity Society, and the European Section of the Cervical Spine Research Society, 27(1), 60-75. https://doi.org/10.1007/s00586-017-5099-2

UK National Institute for Health and Care Excellence. (2016). Low back pain and sciatica in over 16s: assessment and management. https://www.nice.org.uk/guidance/ng59

Urban, J., \& Fairbank, J. (2020). Current perspectives on the role of biomechanical loading and genetics in development of disc degeneration and low back pain; A narrative review. Journal of biomechanics, 102, 109573. https://doi.org/10.1016/j.jbiomech.2019.109573

Verbunt, J. A., Smeets, R. J., \& Wittink, H. M. (2010). Cause or effect? Deconditioning and chronic low back pain. Pain, 149(3), 428-430. https://doi.org/10.1016/j.pain.2010.01.020

Vidal, A. (2017). Lumbalgia la historia interminable. Blog Doloralia, https://www.quironsalud.es/blogs/es/doloralia/lumbalgia-historia-interminable

Videman, T., Nurminen, M., \& Troup, J.D. (1990). Lumbar spinal pathology in cadaveric material in relation to history of back pain, occupation, and physical loading. Spine, 15, 728-740.

Villar, M. (2015). Posturas de trabajo: Evaluación del riesgo. Instituto Nacional de Seguridad e Higiene en el Trabajo.

Vranceanu, A. M., Barsky, A., \& Ring, D. (2009). Psychosocial aspects of disabling musculoskeletal pain. The Journal of bone and joint surgery. American volume, 91(8), 20142018. https://doi.org/10.2106/JBJS.H.01512

Eungpinichpong, W., Buttagat, V., Areeudomwong, P., Pramodhyakul, N., Swangnetr, M., Kaber, D., \& Puntumetakul, R. (2013). Effects of restrictive clothing on lumbar range of motion and trunk muscle activity in young adult worker manual material handling. Applied ergonomics, 44(6), 1024-1032. https://doi.org/10.1016/j.apergo.2013.04.002

Wikström, B., Kjellberg, A., \& Landström, U. (1994). Health effects of long-term occupational exposure to whole-body vibration: A review. International Journal of Industrial Ergonomics, 14, 273-292.

Winkel, J., \& Westgaard, R. H. (1996). A model for solving work related musculoskeletal problems in a profitable way. Applied ergonomics, 27(2), 71-77. https://doi.org/10.1016/0003-6870(95)00061-5

Wong, J. J., Côté, P., Sutton, D. A., Randhawa, K., Yu, H., Varatharajan, S., Goldgrub, R., Nordin, M., Gross, D. P., Shearer, H. M., Carroll, L. J., Stern, P. J., Ameis, A., Southerst, D., Mior, S., Stupar, M., Varatharajan, T., \& Taylor-Vaisey, A. (2017). Clinical practice guidelines for the noninvasive management of low back pain: A systematic review by the Ontario Protocol for Traffic Injury Management (OPTIMA) Collaboration. European journal of pain, 21(2), 201216. https://doi.org/10.1002/ejp.931

Wun, A., Kollias, P., Jeong, H., Rizzo, R. R., Cashin, A. G., Bagg, M. K., McAuley, J. H., \& Jones, M. D. (2021). Why is exercise prescribed for people with chronic low back pain? A review of the mechanisms of benefit proposed by clinical trialists. Musculoskeletal science \& practice, 51, 102307. https://doi.org/10.1016/j.msksp.2020.10230 
Yang, H., Haldeman, S., Lu, M. L., \& Baker, D. (2016). Low back pain prevalence and related workplace psychosocial risk factors: A study using data from the 2010 National Health Interview Survey. Journal of manipulative and physiological therapeutics, 39(7), 459-472. https://doi.org/10.1016/j.jmpt.2016.07.004

Zhang, T. T., Liu, Z., Liu, Y. L., Zhao, J. J., Liu, D. W., \& Tian, Q. B. (2018). Obesity as a risk factor for low back pain: A meta-Analysis. Clinical spine surgery, 31(1), 22-27. https://doi.org/10.1097/BSD.0000000000000468 